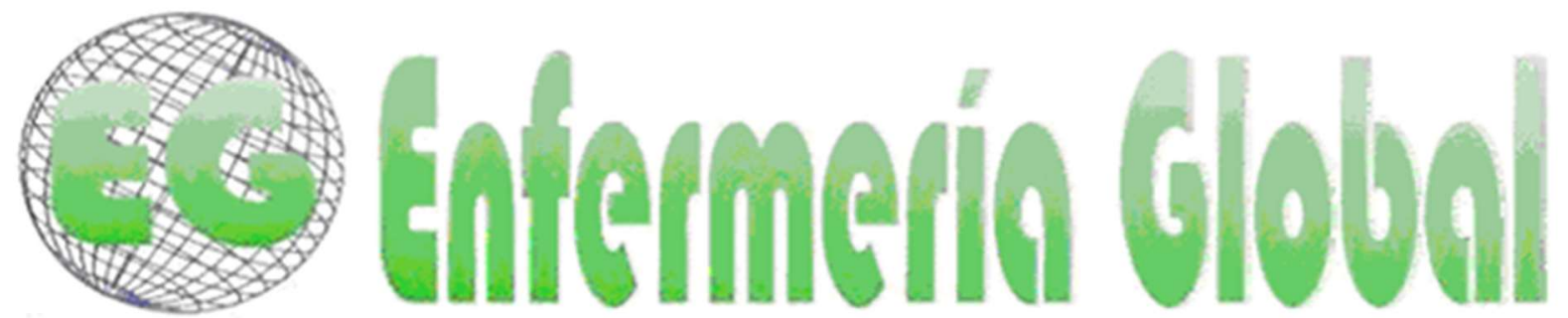

\title{
ORIGINALES
}

\section{Depresión, ansiedad, función cognitiva y dependencia funcional en adultos mayores hospitalizados}

Depression, anxiety, cognitive function, and functional dependence in hospitalized older adults

\section{Tirso Duran-Badillo ${ }^{1}$}

Víctor Alfonso Benítez Rodríguez ${ }^{2}$

Ma de la Luz Martínez Aguilar ${ }^{1}$

Gustavo Gutiérrez Sánchez ${ }^{1}$

Jorge Luis Herrera Herrera ${ }^{3}$

Martha Elba Salazar Barajas ${ }^{1}$

${ }^{1}$ PhD. Profesor de tiempo completo. Unidad Académica Multidisciplinaria Matamoros-Universidad Autónoma de Tamaulipas (UAMM-UAT). México. marthasalbar@yahoo.com.mx

2 Licenciado en Enfermería. Hospital General Dr. Alfredo Pumarejo, Matamoros, Tamaulipas, México.

${ }^{3}$ Doctorando en Enfermería.Universidad del Sinú Elias Bechara Zainum, Colombia.

\section{https://doi.org/10.6018/eglobal.422641}

Recibido: $11 / 04 / 2020$

Aceptado: 19/07/2020

\section{RESUMEN:}

Objetivo: Determinar la relación entre la depresión, ansiedad y función cognitiva con la dependencia en adultos mayores.

Material y Método: Estudio descriptivo y correlacional de corte transversal en 98 adultos mayores hospitalizados. Se aplicó la Escala Hospitalaria de Ansiedad y Depresión, el test de evaluación cognitiva de Montreal e Índice de Barthel.

Resultados: La edad, el género, la depresión, la ansiedad y la función cognitiva explican el 33\% de la dependencia funcional. Las variables que influyen sobre la dependencia en las actividades básicas de la vida diaria fueron el género $(p=.000)$, la depresión $(p=.002)$ y la función cognitiva $(p=.002)$.

Conclusiones: En la valoración de la funcionalidad del adulto mayor hospitalizado es importante considerar el género, la depresión y la función cognitiva.

Palabras clave: depresión; ansiedad; cognición; actividades cotidianas; anciano.

\section{ABSTRACT:}

Objective: Determine the relationship between depression, anxiety, and cognitive function with dependency in older adults.

Materials and Method: Descriptive and correlational cross-sectional study conducted on 98 hospitalized older adults. The Hospital Anxiety and Depression Scale, the Montreal Cognitive Evaluation Test, and the Barthel Index were applied.

Results: Age, gender, depression, anxiety, and cognitive function explain $33 \%$ of functional dependency. The variables that influence dependence to carry out basic activities in daily life were 
gender $(p=.000)$, depression $(p=.002)$, and cognitive function $(p=.002)$.

Conclusions: In assessing the functionality of hospitalized older adults, gender, depression, and cognitive function are important to consider.

Key words: Depression; anxiety; cognition; daily life activities; older adult.

\section{INTRODUCCIÓN}

El aumento de la esperanza de vida y el control de la natalidad han dado como resultado incremento de la población (1). El vivir más representa un reto para las personas, porque significa mantenerse activo en las mejores condiciones físicas, emocionales y psicológicas. Es decir, para la persona mayor implica desafíos personales, familiares, económicos y sociales.

A mayor edad, mayor es el riesgo de padecer enfermedades, y el padecer una o más enfermedades, aumenta la probabilidad de ser hospitalizado, y provocar con ello estados de ansiedad y depresión, alteración cognitiva y dependencia funcional. Durante la hospitalización se han comprobado prevalencias de síntomas de ansiedad y síntomas depresivos de 40 a $50 \%{ }^{(2)}$.

La depresión y ansiedad son trastornos mentales muy frecuentes (3) y la evidencia científica muestra que los pacientes hospitalizados en cuidados intensivos presentan cifras de ansiedad y depresión de 20 a $30 \%{ }^{(4)}$ en medicina interna de $22 . \%$ y $28 . \%$ respectivamente ${ }^{(5)}$. Los síntomas depresivos afectan a más mujeres con dependencia funcional para las actividades básicas de la vida diaria y la diabetes mellitus es la enfermedad que más comúnmente ha sido asociada a síntomas depresivos ${ }^{(6)}$ y Otros autores mencionan cifras de $57 \%$ en este mismo padecimiento ${ }^{(7)}$.

Por otro lado, el proceso que viven las personas mayores durante la hospitalización y su falta de capacidad para adaptarse (8) puede influir en su función cognitiva, la disminución de la comunicación, la separación de seres queridos, el tratamiento, el espacio físico, las condiciones del aire, la iluminación, los olores, son situaciones que se presentan y probablemente influyen de forma negativa, y favorecen, las condiciones para incrementar los estados de ansiedad, depresión, y dependencia funcional (9).

La dependencia funcional, ha sido definida como "el estado en el que se encuentran las personas que, por razones ligadas a la falta o pérdida de autonomía física, psíquica o intelectual, tienen necesidad de asistencia o ayuda importante para realizar las actividades de la vida cotidiana". La literatura muestra que la actividad física podría ser protectora de las facultades cognitivas y esta a su vez es necesaria para mejorar la calidad de vida ${ }^{(10)}$ produciendo un círculo agravante, donde los trastornos emocionales pueden ser tan inhabilitantes como cualquier otra enfermedad física.

El Instituto Nacional de Salud mental, menciona que las condiciones de temor, la capacidad limitada de confiar en los demás, la alteración en las relaciones de familia, durante la hospitalización, hacen que exista mayor vulnerabilidad para presentar riesgo de complicaciones no relacionadas a la enfermedad de ingreso, y quizá puedan prolongar la estancia hospitalaria (11). El estar en cama por tiempo prolongado y disminuir la actividad física, condiciona a presentar factores de riesgo en el sistema cardiovascular, respiratorio y músculo esquelético. 
La literatura muestra que el $33 \%$ de los adultos mayores hospitalizados presentarán deterioro funcional en al menos una de las actividades de la vida diaria y se incrementa hasta un $50 \%$ cuando superan la edad de 80 años (12). A pesar de la relevancia en materia de salud, de las variables depresión, ansiedad, síntomas depresivos y deterioro funcional en adultos mayores hospitalizados, no se ha encontrado suficiente evidencia científica que ayude a formular estrategias para identificar condiciones de salud en la población (13).

Los profesionales de enfermería por su ubicación en el ámbito hospitalario y el cuidado asistencial pueden contribuir a detectar de forma precoz los primeros síntomas de ansiedad y depresión, la alteración en la función cognitiva en las personas adultas hospitalizadas.

Por tal razón el objetivo fue determinar la relación entre la depresión, ansiedad y función cognitiva con la dependencia en adultos mayores. Se pretende que los resultados del presente trabajo incrementen el cuerpo de conocimientos de enfermería, oriente a intervenciones para identificar y mejorar la situación emocional, evitar la pérdida de funciones en el adulto mayor hospitalizado y de ésta manera las instituciones de salud se verán beneficiadas al disminuir el tiempo de hospitalización y las personas mayores tendrán una mejor calidad de vida.

\section{METODOLOGÍA}

El diseño del estudio fue de tipo descriptivo y correlacional de corte transversal, se realizó en la cd. De Matamoros, Tamaulipas (México). La población estuvo compuesta por adultos mayores de 60 años que ingresen a hospitalización en un Hospital General de Matamoros, Tamaulipas. El muestreo no probabilístico, por conveniencia, se incluyeron en el estudio a 98 adultos mayores que ingresaron a hospitalización en el tiempo establecido para la recolección de datos. Se incluyeron como adultos mayores a personas con 60 años y más que ingresaron a hospitalización en las salas de medicina interna, cirugía y ginecoobstetricia, durante el segundo semestre del 2018.

Para la recolección de los datos se utilizó una cédula de datos personales para el registro de datos sociodemográficos y de salud. Para medir la ansiedad y depresión se utilizó la Escala Hospitalaria de Ansiedad y Depresión ${ }^{(14)}$, contiene 14 ítems, siete miden la ansiedad y siente la depresión. La opción de respuesta es en escala tipo Likert que oscilan del cero al tres. Para el análisis se hace una sumatoria por subescala, con un puntaje mínimo de cero y máximo de 21. Los puntos de corte de cero a siete indican normal, de ocho a diez sugiere caso probable de ansiedad y/o depresión y del 11 al 21 caso de ansiedad y/o depresión, se ha reportado un Alpha de Cronbach del .88 al .90 para población mexicana ${ }^{(14)}$.

Para medir el deterioro cognitivo se utilizó el test de evaluación cognitiva de Montreal (MoCA $^{(15)}$, los reactivos y puntajes son: nivel visuespacial/ejecutiva (5 puntos), identificación (3 puntos), atención (6 puntos), lenguaje (3 puntos), abstracción (2 puntos), recuerdo diferido (5 puntos) y orientación (6 puntos). Se deben sumar los puntos obtenidos en cada una de las habilidades evaluadas, un puntaje igual o superior a 26 corresponde a un individuo normal, y un puntaje inferior lo clasifica con 
deterioro cognitivo leve, cabe señalar que el instrumento sugiere sumar un punto a la sumatoria final a aquellos sujetos que reportan menos de 12 años de escolaridad ${ }^{15}$. El Alpha de Cronbach reportado para la versión en español en adultos mayores es de $.77^{(16)}$

La dependencia funcional se midió con el índice de Barthel ${ }^{(17)}$, mide 10 Actividades Básicas de de la Vida Diaria (ABVD), a la manera en como realizan sus actividades se les asigna una puntuación que oscila de cero a quince puntos, finalmente se hace una sumatoria que va de 0 a 100 puntos. Los resultados globales se agrupan en cuatro categorías de dependencia, se consideraron las sugeridas por Shah, Vanclay y Cooper: 17 1) < 21 puntos dependencia total, 2) de 21 a 60 puntos dependencia severa, 3) de 61 a 90 puntos dependencia moderada, 4) de 91 a 99 puntos dependencia escasa y, 5) 100 puntos independencia.

El estudio fue aprobado por el comité de ética e investigación de la Unidad Académica Multidisciplinaria Matamoros de la Universidad Autónoma de Tamaulipas, (Registro 050) las consideraciones del estudio incluyeron las recomendaciones del Reglamento de la Ley General de Salud en materia de investigación, el respeto a la dignidad y el derecho de los participantes, la privacidad y el consentimiento informado.

El análisis de los datos se realizó con el paquete estadístico IBM SPSS versión 21 para Windows. Se utilizó estadística descriptiva a través de frecuencias y proporciones que permitieron describir los aspectos sociodemográficos de los participantes. La distribución de las variables con la prueba de bondad de ajuste de Kolmogorov-Smirnov con corrección de Lilliefors, y en función de los resultados se optó por utilizar estadística no paramétrica (Correlación de Spearman y U de ManWhitney). Se realizó un modelo lineal general de contraste univariado donde las variables depresión, ansiedad y función cognitiva se ubicaron como variables independientes y la dependencia en ABVD como variable dependiente.

\section{RESULTADOS}

Se evaluaron 98 adultos mayores hospitalizados con edad promedio de 66.30 años $(D E=6.382)$, el $55.1 \%(f=54)$ mujeres, el $55.1 \%(f=54)$ sin pareja marital. El $42.9 \%$ $(f=42)$ no presentó signos de depresión, el 30.6\% $(f=30)$ con probable depresión y el $26.5 \%(f=26)$ con depresión establecida. El 53.1\% ( $f=53)$ no presentó signos de ansiedad, el $33.7 \%(f=33)$ con probable ansiedad y el $13.3 \%(f=13)$ con ansiedad establecida.

El 60.2\% ( $f=59)$ resultó con deterioro cognitivo. En las ABVD el $8.2 \%(f=8)$ con dependencia total, el $26.5 \%(f=26)$ dependencia severa, el $34.7 \%(f=34)$ con dependencia moderada, el $4.1 \%(f=4)$ dependencia escasa y el $26.5 \%(f=26)$ con independencia. En la tabla 1 se encuentran otros datos descriptivos de la depresión, ansiedad, función cognitiva y dependencia funcional. 
Tabla 1. Datos descriptivos de la depresión, ansiedad, función cognitiva y dependencia funcional.

\begin{tabular}{lcccc}
\hline Variable & Min & Max & Media & DE \\
\hline Edad & 60 & 92 & 66.30 & 6.382 \\
Depresión & 0 & 17 & 8.13 & 3.732 \\
Ansiedad & 0 & 14 & 7.08 & 3.105 \\
Función cognitiva & 0 & 30 & 23.02 & 5.962 \\
Dependencia funcional & 1 & 100 & 69.69 & 27.487 \\
\hline
\end{tabular}

La prueba de correlación de Spearman mostró que la edad se asocia positivamente con la depresión $\left(r_{\mathrm{s}}=.264 ; p=.009\right)$ y la ansiedad $\left(r_{\mathrm{s}}=.245 ; p=.015\right)$ y negativamente con la función cognitiva $\left(r_{s}=-258 ; p=.010\right)$ y la dependencia funcional $\left(r_{s}=-.225\right.$; $p=.026)$. En la tabla 2 se observa que la depresión $(p=.000)$, ansiedad $(p=.011)$ y función cognitiva $(p=.001)$ se relacionaron significativamente con la dependencia funcional para realizar las actividades de la vida diaria.

Tabla 2. Relación entre depresión, ansiedad y función cognitiva con la dependencia funcional

\begin{tabular}{lcc}
\hline Variable & \multicolumn{2}{c}{ Dependencia Funcional } \\
\cline { 2 - 3 } & $\mathrm{r}_{\mathrm{s}}$ & $p$ \\
\hline Depresión & -.419 & .000 \\
Ansiedad & -.256 & .011 \\
Función cognitiva & .319 & .001 \\
\hline
\end{tabular}

Se realizó un análisis de regresión lineal para conocer las variables que influyen en la dependencia funcional. Las variables independientes, edad, género, estado marital, depresión, ansiedad y función cognitiva explica el $33 \%$ de la dependencia funcional $\left[F(6,97)=9.031, p<.001 ; R^{2}=.33\right]$. Las variables significativas en el modelo fueron el género, depresión y ansiedad (Tabla 3 ).

Tabla 3. Influencia de variables personales, depresión, ansiedad y función cognitiva sobre la dependencia funcional

\begin{tabular}{llllll}
\hline Variable & $\mathrm{SC}$ & $\mathrm{g}^{1}$ & $\mathrm{CM}$ & $\mathrm{FI}$ & $p$ \\
\hline Edad & .805 & 1 & .805 & .002 & .968 \\
Género & 7269.48 & 1 & 7269.486 & 14.401 & .000 \\
Estado marital & 364.39 & 1 & 364.390 & .722 & .398 \\
Depresión & 5021.43 & 1 & 5021.434 & 9.947 & .002
\end{tabular}




$\begin{array}{llllll}\text { Ansiedad } & 11.55 & 1 & 11.557 & .023 & .880 \\ \text { Función cognitiva } & 5354.65 & 1 & 5354.659 & 10.607 & .002\end{array}$

Dependencia funcional: $\mathrm{R}$ ajustado=.33

\section{DISCUSIÓN}

La caracterización demográfica realizada en la presenta investigación, guarda estrecha relación con los datos reportados por el Banco Interamericano de Desarrollo (BID), en su informe sobre el Panorama de envejecimiento y dependencia en América latina y el Caribe, el cual reporta similares variables y otras condiciones que dan cuenta de un contexto que establece que el $70 \%$ de los años de vida perdidos por muerte prematura e incapacidad en esta región es atribuible a enfermedades cardiovasculares, seguido de los trastornos neurodegenerativos como el Alzheimer y la demencia senil ${ }^{(18)}$, mismas condiciones que generan grados variables de dependencia y síntomas físicos ${ }^{(19)}$ y psicológicos con un impacto significativo en la calidad de vida ${ }^{(20)}$.

Los resultados de la prueba de correlación mostraron que, a mayor edad mayor depresión y mayor ansiedad, lo cual difiere con lo encontrado por Hernández y colaboradores $^{(21}$, quienes al evaluar a un grupo de adultos mayores institucionalizados, no encontraron relación entre la edad y la presencia de depresión. En este sentido, al revisar la literatura disponible no se encuentra evidencia que permita evaluar relación significativa entre la edad y trastornos de tipos psicológico como la ansiedad; sin embargo, la edad si es una variable de la cual se ha demostrado estar asociada a trastornos como las morbilidades ${ }^{(22,23)}$.

Por otra parte, al evaluar la asociación entre edad y deterioro cognitivo, se encontró que a mayor edad, mayor deterioro cognitivo; situación que es respaldada por resultados en países como Colombia, en donde Camargo et al., (24) establecieron que $41,7 \%$ de los adultos con edad superior a los 60 años, presentaron deterioro cognitivo grave. Así mismo, revisiones de la literatura; lograron determinar grados variables de deterioro cognitivo moderado que dependían de los grupos de edad estudiados (25); lo que permite establecer relación entre esta variable y la prevalencia de dicha condición.

Así mismo, la literatura documenta un aumento de la dependencia funcional con el proceso de envejecimiento, siendo más marcada en la medida que se ganan más años de vida; tal como lo reporto Laguado et al. ${ }^{(26)}$, en su investigación en adultos mayores institucionalizados en centros de bienestar, quien estableció algún grado de dependencia en los mayores de 60 años, resultado similar a los encontrados en la presente investigación, la cual encontró que a mayor edad mayor dependencia funcional.

Cuando se analizó, la relación entre la presencia de condiciones como depresión y ansiedad en los adultos mayores y la función cognitiva; con la dependencia, se tuvo que: a mayor depresión mayor dependencia funcional, de igual forma, a mayor ansiedad mayor dependencia funcional y a menor función cognitiva mayor grado de dependencia. Relación, que ha sido abordada a la hora de estudiar la salud mental 
del adulto mayor y que demuestra una asociación positiva, tal como lo deja ver un estudio en España que demostró que los niveles altos depresión, ansiedad y el deterioro cognitivo demostraron una correlación positiva con la dependencia funcional(27). Lo que se iguala a lo obtenido en esta investigación.

Por su parte, el modelo de regresión lineal mostró que las variables edad, género, estado marital, depresión, ansiedad y función cognitiva influyen en la dependencia funcional de los adultos mayores hospitalizados; situación variable en diferentes investigaciones, en el sentido de que algunos han encontrado relación estadísticamente significativa entre estas y la dependencia ${ }^{(20)}$, mientras que otras difieren en estos resultados.

\section{CONCLUSIONES}

En relación al fenómeno de dependencia funcional en los adultos mayores, esta investigación, muestra relación positiva entre este y variables demográficas como la edad, género y estado marital, así como con variables de tipo biológico como el deterioro cognitivo.

Lo anterior aporta elementos importantes, en el marco del proceso de atención de esta población, toda vez que existe una pluralidad de resultados que si bien es cierto dificultad la unificación de criterios, permite vislumbrar un escenario que requiere de un mayor abordaje desde la investigación, que den cuenta de elementos que puedan ser retomados por los tomadores de decisiones con el fin de garantizar intervenciones enfocadas a un envejecimiento saludable.

Teniendo en cuenta, desde una perspectiva biológica, que el proceso de envejecimiento incluye una reducción significativa de las capacidades no solo físicas, sino también mentales, que aumentan de manera exponencial el riesgo de enfermar.

Para finalizar, las proyecciones realizadas por organismos internacionales como la Organización Mundial de la Salud, en relación con el envejecimiento de la población, se ha cumplido; observándose una población adulta mayor, con una creciente demanda de servicios sanitarios y de cuidado. Lo que se convierte en un punto de interés para los equipos multidisciplinario de atención salud.

\section{REFERENCIAS}

1. Organización mundial de la salud. Envejecimiento y ciclo de vida. 2018. Disponible en: https://www.who.int/ageing/about/facts/es/.

2. Anguiano-Serrano SA, Mora-Miranda MA, Reynoso-Erazo L,Vega-Valero CZ. Eureka:Asunción (Paraguay). 2017; 14(1):24-38. ISSN 2218-0559 (CD R), EISSN2220-9026.

3. Organización Mundial de la Salud. Salud mental. 2017. Disponible en: https://www.who.int/mental health/es/.

4. Costas -González M, Prado-Robles V, Crespo - Iglesias JM. Ansiedad y depresión entre los pacientes hospitalizados en el complejo hospitalario de Ferrol. C. Med. Psicocom. 2013; 107 (1):16-22. 
5. Von- Mühlenbrock SF, Gómez DR, González VM, Rojas AA, Vargas GL, VonMühlenbrock PC. Late life depression prevalence in patients admitted to an Internal Medicine Service. Rev. chil. neuro-psiquiatr. [Internet]. 2011 [citado 2018 Oct 19]; 49(4): 331-337. Disponible en: http://dx.doi.org/10.4067/S0717-92272011000400004.

6. Prieto-Miranda SE, Villanueva -Muñiz EY, Arias -Ponce N, Jiménez-Bernardino CA. Depresión de inicio tardío en el adulto Mayor hospitalizado en un hospital de segundo nivel. Med Int México. 2016; 32(4) 338-395. https://web.a.ebscohost.com/abstract?direct

7. Constantino-Cerna A, Bocanegra-Malca M, León-Jiménez F, Díaz-Vélez C. Frecuencia de depresión y ansiedad en pacientes con diabetes tipo 2 atendidos en un hospital general de Chiclayo. Rev Med Hered. 2014; 25(4): 196-203. Disponible en: http://www.scielo.org.pe/scielo.php?script=sci arttext\&pid=S1018130X2014000400003\&lng=es.

8. Ocampo-Chaparro JM, Mosquera-Jiménez JI, Davis SA, Reyes-Ortiz CA. Deterioro funcional asociado al deterioro cognitivo en el anciano hospitalizado. Rev Esp Geriatr Gerontol. 2018; 53(1): 19-22.

9. Helvik AS, Engedal K, Selbaek G. Perceived health among the medically hospitalized elderly: A one-year follow-up study. Scand J public Health.2013. 41(4): 421-8 https://journals.sagepub.com/doi/10.1177/1403494813480278

10. Alomoto MM, Calero MS, Vaca GM. Intervention with physical-recreational activity to anxiety and depression in the elderly. Revista Cubana de Investigaciones Biomédicas. 2018; 37(1), 47-56.

11. Zas Ros B. Experiencias en Psicologia hospitalaria. Editorial ALFEPSI. La organización de los servicios psicológicos en los hospitales. 2016; (p:27) IBN 978607-96454-3-4

12. Ibarra- Cornejo JL, Fernández -Lara MJ, Aguas- Alveal EV, Pozo- Castro AF, Antillanca-Hernández B, Quidequeo -Reffers DG. Efectos del reposo prolongado en adultos mayores hospitalizados. Anales de la Facultad de Medicina. 2017; 78(4), 439 444.

13. Encuesta Nacional de Salud y Nutrición -ENSANUT- [Internet]. 2016 [cited 3 May 2018]. Disponible en: http://promocion.salud.gob.mx/dgps/descargas1/doctos_2016/ensanut_mc_2016310oct.pdf.

14. Yamamoto-Furusho JK, Sarmiento-Aguilar A, Garcia-Alanis M, Gómez-Garcia, L E, Toledo-Mauriño J, Olivares-Guzmán L, Fresán-Orellana A. Escala de Ansiedad y Depresión Hospitalaria (HADS): Validación en pacientes mexicanos con enfermedad inflamatoria intestinal. Gastroenterología y Hepatología. 2018; 41(8), 477-482.

15. Lozano M, Hernández M, Turró O, Pericot I, López-Pousa S, Vilalta J. Validación del Montreal Cognitive Assessment (MoCA): test de cribado para el deterioro cognitivo leve. Datos preliminares. Alzheimer Realidades e Investigación en Demencia. 2009; 43, 4-11

16. Delgado C, Araneda A, Behrens M I. Validación del instrumento Montreal Cognitive Assessment en español en adultos mayores de 60 años. Neurología. 2019; 34(6), 376-385.

17. Shah S, Vanclay F, Cooper B. Improving the sensitivity of the Barthel Index for stroke rehabilitation. Journal of Clinical Epidemiology. 1989; 42 (8), 703-709.

18. Aranco N, Stampini M, Ibarrarán $P$, Medellín N. Panorama de envejecimiento y dependencia en América Latina y el Caribe. Resumen de Políticas. Washington DC, Estados Unidos. 2018.

19. Cerquera A, Uribe A, Matajira Y, Correa H. Dependencia funcional y dolor crónico 
asociados a la calidad de vida del adulto mayor. Psicogente. 2017; 20 (38), 398-409. http://doi.org/10.17081/psico.20.38.2561

20. Paredes- Arturo YV, Yarce- Pinzón E, Aguirre- Acevedo DC. Funcionalidad y factores asociados en el adulto mayor de la ciudad San Juan de Pasto, Colombia. Rev Cienc Salud. 2018;

Doi:http://dx.doi.org/10.12804/revistas.urosario.edu.co/revsalud/a.6494. 16(1):114-128.

21. Hernández L, Muñiz N, Gutiérrez M, Hernández J, Chan M,Cetina F. Depresión en un centro geriátrico comunitario en Quintana Roo. Revista salud Quintana Roo. 2016; 9(35), 6-10.

22. Casas-Rojas EC, Cardona-Arango D, Segura-Cardona Á, Garzón-Duque M O. Multimorbilidad auto-reportada por el adulto mayor del departamento de Antioquia: prevalência y factores asociados. CES Medicina. 2018; 32(2), 116-128. https://dx.doi.org/10.21615/cesmedicina.32.2.5

23. Morales Erazo A, Rojas G M, Pinzón E Y, Paredes -Arturo Y V, Rosero- Otero M, Hidalgo A. Condiciones médicas prevalentes en adultos mayores de 60 años. Acta Médica Colombiana. 2016; 41(1), 21-28. https://doi.org/10.36104/amc.2016.615

24. Camargo-Hernández K C, Laguado-Jaimes E. Grado de deterioro cognitivo de los adultos mayores institucionalizados en dos hogares para ancianos del área metropolitana de Bucaramanga - Santander, Colombia. Rev Univ. Salud. 2017; 19(2):163-170. DOI: http://dx.doi.org/10.22267/rus.171902.79

25. Benavides-Caro C A.Deterioro cognitivo en el adulto mayor. Revista Mexicana de Anestesiología 2017; 40(2), 107-112.

26. Laguado Jaimes E, Camargo Hernández K C, Campo Torregroza E, Martín Carbonell MC.Funcionalidad y grado de dependencia en los adultos mayores institucionalizados en centros de bienestar. Gerokomos. 2017; 28(3), 135-141.

27. Rodríguez Díaz MT, Cruz-Quintana F, Pérez-Marfil MN. Dependencia funcional y bienestar en personas mayores institucionalizadas. Index de Enfermería. 2014; 23(12), 36-40.

ISSN 1695-6141

๑ COPYRIGHT Servicio de Publicaciones - Universidad de Murcia 